\title{
Four-way Waveguide Power Dividers with Integrated Filtering Function
}

\author{
Ali Musa Mohammed \\ Electrical, Electronic and Computer Engineering \\ University of Greenwich (Medway Campus) \\ Chatham, ME4 4TB, U.K. \\ Electrical and Electronic Engineering Technology \\ The Federal Polytechnic Damaturu, Nigeria
}

\author{
Yi Wang \\ Electrical, Electronic and Computer Engineering \\ University of Greenwich (Medway Campus) \\ Chatham, ME4 4TB, U.K. \\ yi.wang@gre.ac.uk
}

\begin{abstract}
A novel power divider with filtering function has been proposed in this work. A coupled-resonator structure is utilized to realise the dual functions. This eliminates the need for conventional transmission-line based splitting networks. The corresponding coupling matrix has been synthesised. A four-way filtering power divider was designed with an equal power division at $\mathrm{X}$-band using rectangular waveguide cavities. A return loss of $20 \mathrm{~dB}$ and a bandwidth of $500 \mathrm{MHz}$ with a fourthorder Chebyshev filtering response were demonstrated. The design has been verified by measurements, which show good agreements with simulations.
\end{abstract}

Keywords- Filters, power dividers, coupling matrix, waveguides

\section{INTRODUCTION}

Power divider is used in many microwave circuits to divide or combine the power of microwave signals. Conventional power dividers are transmission-line based with very limited frequency selectivity. When high selectivity or rejection is required to suppress unwanted signals, bandpass filters are usually added in a cascaded form. Since both components - the power divider and the filter - occupy a relatively large footprint in the front-ends, it becomes very important to find ways to integrate the two components seamlessly and reduce their sizes. An integrated filtering power divider will also have the advantage of reduced insertion losses and inter-component mismatches. Several techniques have been proposed in the last few years to achieve such a device integration over the conventional cascaded system. One simple way to increase the selectivity of a power divider is to add stubs, creating transmission notches [1]. A more sophisticated and effective way is to use resonator based multi-port filtering networks to realize power dividers [2] - [8]. Various types of resonators microstrip [2] - [4] and waveguide [5] - were utilized to realize different frequency responses such as Chebyshev or quasielliptic in three-port power dividers. A combination of half and quarter-wavelength resonators were used to achieve highharmonic rejection [3]. In [6], a four-port $180^{\circ}$ hybrid with a third-order Chebyshev filtering response was realized by transformation into a coupled resonator network. Gysel power dividers with bandpass responses were also demonstrated [7], [8]. All the above-mentioned are two-way dividers. To use power dividers in antenna arrays or multiport amplifiers, more power outputs are often required.

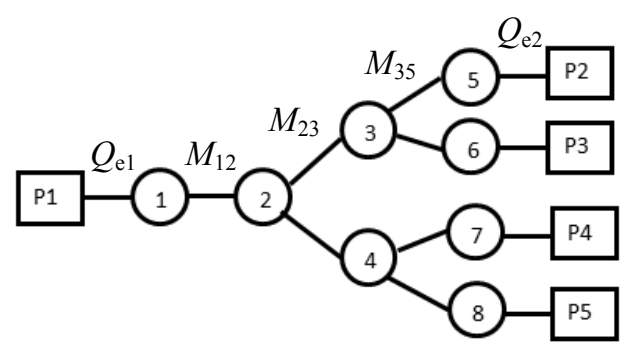

(a)

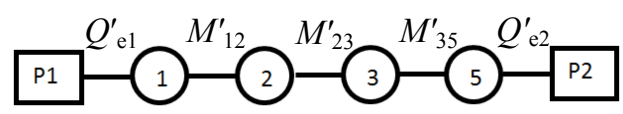

(b)

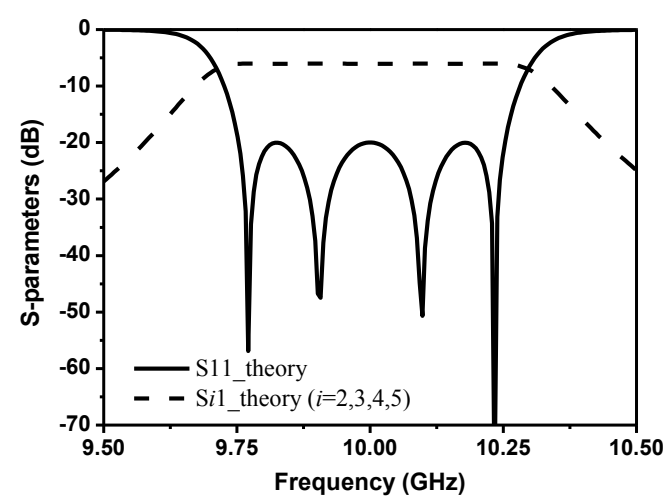

(c)

Fig. 1. (a) Topology of the proposed power divider; (b) A filter of the same order as the power divider; (c) Frequency domain responses from the synthesized coupling matrix of the power divider.

In this paper, a four-way filtering power divider was proposed based on a bifurcated coupled-resonator structure as shown in Fig. 1(a). A similar topology has been used in a fourchannel multiplexer [9]. X-band rectangular waveguide cavities were utilized to demonstrate the filtering power divider. The design started with the acquisition of the required coupling matrix. This has been achieved by either direct synthesis or optimization. This paper is organized as follows: 
Section II presents the synthesis of the coupling matrix for the coupled-resonator structure. Section III describes the design and implementation using the waveguide technology. Simulation and measurement results are presented and discussed in Section IV, which is followed by the conclusion in Section V.

\section{SYNTHESIS OF MULTI-PORT COUPLING MATRIX}

Coupling matrix is an important means of design and analysis of coupled-resonator filters. It has been shown that this means can be extended from two-port to multi-port filtering network formed of coupled resonators [5], [9], [10]. For a multi-port resonator network with $N$ coupled resonators, the general normalized coupling matrix $[A]$ in terms of normalized coupling coefficient and external quality factors can be expressed in (1).

$$
[A]=[r]+p[U]-j[m]
$$

where $U$ is the $N \times N$ identity matrix and $[m]$ is the general coupling matrix. $[r]$ is an $N \times N$ matrix with all entries zero except for $[r]_{i i}=1 / q_{e i}$, where $i$ is the index of the resonators attached to the port. In the case of the four-way power divider concerned here, $N=8$ and $i=1,5,6,7,8 . p$ is the complex low-pass frequency variable, $p=j\left(\omega / \omega_{0}-\omega_{0} / \omega\right) / F B W$. $F B W$ is the fractional bandwidth. After transformation into the real frequency domain, the external quality factors and the coupling matrix become

$$
Q_{e i}=q_{e 1} / F B W, \quad M_{i j}=m_{i j} \cdot F B W .
$$

The scattering parameters in a multi-port network with $N$ coupled resonators can be related to the matrix $[A]$ in the following form in (3).

$$
\begin{aligned}
& S_{m m}=1-\frac{2}{q_{e i}}[A]_{i i}^{-1} \\
& \left.S_{m n}\right|_{m \neq n}=\frac{2}{\sqrt{q_{e i} q_{e j}}}[A]_{i j}^{-1}
\end{aligned}
$$

where $m$ and $n$ are the indices of the ports whereas $i$ and $j$ are the indices of the resonators. So $S_{m m}$ is the reflection coefficient at the port $P m$, attached to the $i$-th resonator. $S_{m n}$ is the transmission coefficient from the port $P n$ to $P m$, which are attached to the $j$-th and $i$-th resonators respectively.

The four-way power divider contains eight resonators. As depicted in Fig. 1(a), each circle represents a resonator. The couplings between resonators are $M_{\mathrm{ij}}$ and the external couplings are represented by $Q_{e i}$. An arbitrary bifurcated topology is generally not directly synthesisable, when a local optimisation based method can be used to synthesize its coupling matrix. The cost function for the optimization assesses the deviations from at least three specified target parameters. These include the level of the return loss across the passband as well as at the band edges which is $20 \mathrm{~dB}$ in this case, and the number of reflection zeros in the passband which is four. Polynomials of the transfer functions are attainable but not required in the synthesis.
Due to the high-symmetry of the concerned equal power divider in this work, the coupling matrix can be directly acquired by comparison with a two-port filter of the same order (fourth order, as shown in Fig. 1b) as the divider. Simple circuit analysis using inverters shows that to achieve a fourthorder Chebyshev response requires

$$
\begin{aligned}
& M_{12}=M_{12}^{\prime}, Q_{e i}=Q_{e i}^{\prime}, \\
& M_{23}=M_{23}^{\prime} / \sqrt{2}, M_{35}=M_{35}^{\prime} / \sqrt{2},
\end{aligned}
$$

where $M_{\mathrm{ij}}^{\prime}$ and $Q^{\prime}{ }_{e i}$ are from the coupling matrix of a fourthorder Chebyshev filter. The coupling coefficient $M_{\mathrm{ij}}$ and the external quality factor $Q_{e i}$ of the power divider for a $F B W$ of 5 $\%$ are found to be:

$$
\begin{gathered}
M_{12}=0.0455, M_{23}=M_{24}=0.0247 \\
M_{35}=M_{36}=M_{47}=M_{48}=0.0322, \\
Q_{\mathrm{e} 1}=Q_{\mathrm{e} 5}=Q_{\mathrm{e} 6}=Q_{\mathrm{e} 7}=Q_{\mathrm{e} 8}=18.67
\end{gathered}
$$

Fig. 1(c) shows the theoretical scattering parameters corresponding to the above coupling coefficients.

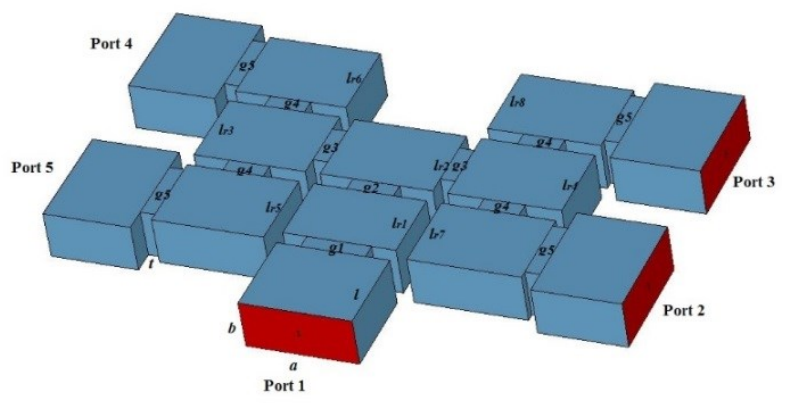

Fig. 2. Design A layout. $a=22.86, b=10.16, l=18.00, l_{\mathrm{r} 1}=14.58, l_{\mathrm{r} 2}=$ $16.56, l_{\mathrm{r} 3}=l_{\mathrm{r} 4}=16.64, l_{\mathrm{r} 5}=l_{\mathrm{r} 6}=l_{\mathrm{r} 7}=l_{\mathrm{r} 8}=16.75, g_{1}=13.33, g_{2}=9.52, g_{3}=9.42$, $g_{4}=8.29, g_{5}=13.79, t=3.00$. All dimensions are in millimetres.

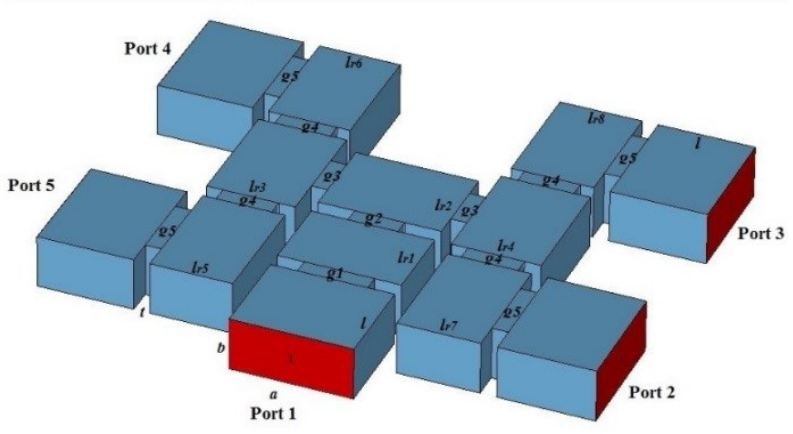

Fig. 3. Design B layout. $a=22.86, b=10.16, l=18.00, l_{\mathrm{r} 1}=14.90, l_{\mathrm{r} 2}=$ $16.91, l_{\mathrm{r} 3}=l_{\mathrm{r} 4}=16.57, l_{\mathrm{r} 5}=l_{\mathrm{r} 6}=l_{\mathrm{r} 7}=l_{\mathrm{r} 8}=15.35, g_{1}=12.96, g_{2}=9.27, g_{3}=8.57$, $g_{4}=10.42, g_{5}=12.99, t=3.00$. All dimensions are in millimetres.

\section{DESIGN}

The proposed power divider in Fig. 1(a) has been implemented using rectangular waveguide cavities with 
inductive couplings. The central frequency is designed to be at $10 \mathrm{GHz}$. WR-90 waveguides are used with $a=22.86 \mathrm{~mm}$ and $b=10.16 \mathrm{~mm}$. Two layouts were investigated (design A and B) as shown in Fig. 2 and Fig. 3 respectively. In design A, all the resonators are laid horizontally along the broad wall of the waveguides whereas in design $\mathrm{B}$ the resonators 3-8 are vertically laid. Computer Simulation Technology (CST) Microwave studio [11] was utilized to determine the initial dimensions of the cavities as well as the coupling irises between neighboring resonators in order to achieve the required coupling $M_{\mathrm{ij}}$ and $Q_{\mathrm{ei}}$ [12]. After interconnecting all the resonators and irises together, due to loading effects the initial dimensions obtained had to be tuned to achieve the desire responses. The variations in dimensions before and after full-wave optimisation were determined. The lengths of the cavities decrease from initial values by about $10 \%$ whereas an increase of about $15 \%$ was recorded for the inductive coupling irises. High structural symmetry was enforced on the design in order to reduce the number of optimization parameters. The dimensions obtained after optimization were given in the captions of Fig. 2 and Fig. 3 for design A and B respectively.

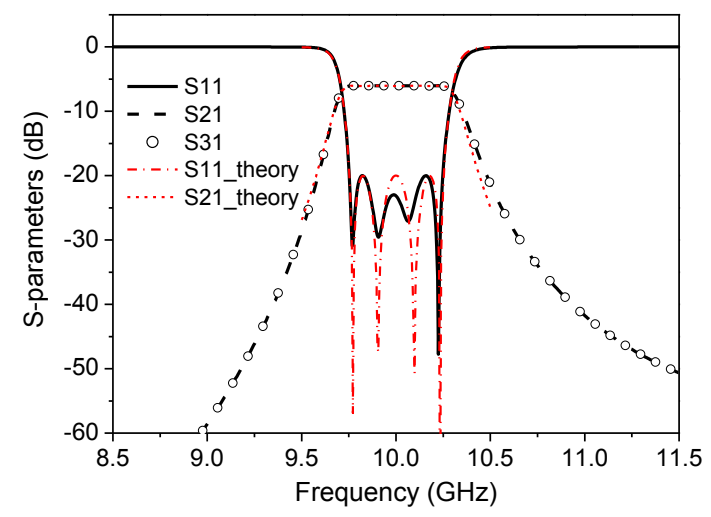

(a)

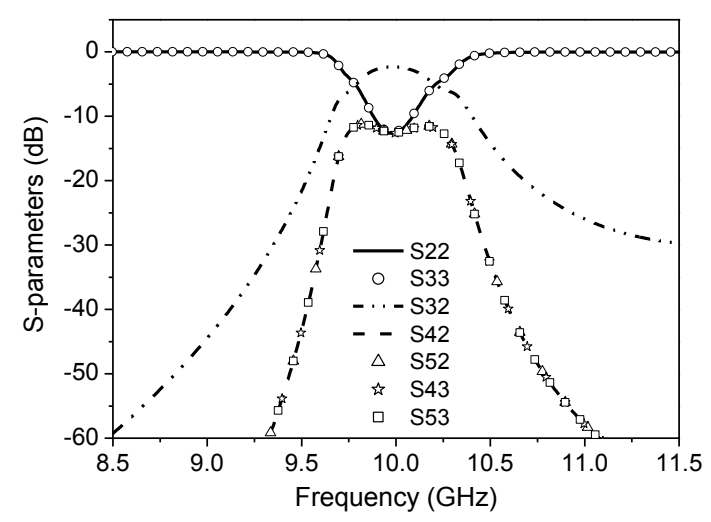

(b)

Fig. 4. Simulated performance of the design B. (a) Input matching and transmission responses in comparison with the theoretical ones; (b) Output matching and inter-port isolations.

The design $\mathrm{B}$ has been optimized to the design specification. As shown in Fig. 4(a), the simulated results show excellent agreement with the theoretical responses derived directly from the coupling matrix. Conductor loss was excluded from the simulation. A fourth-order Chebyshev filtering response with a return loss of $20 \mathrm{~dB}$ is shown. The bandwidth was $500 \mathrm{MHz}$. As expected, the output matching and inter-port isolation were poor without any extra resistive or non-reciprocal circuits in place. As shown in Fig. 4(b), the lowest isolation is between the ports on the same side of the input such as port- 2 and 3 , which is only $2.3 \mathrm{~dB}$. The isolation between the output ports on different sides of the input was $11.2 \mathrm{~dB}$. The isolation level was affected by the number of resonators along the shortest path between ports. The output port matching was $-12.5 \mathrm{~dB}$ at $10 \mathrm{GHz}$. In the simulation model, sharpe corners were assumed. The effect of rounded corners as a result of practical machining was investigated. It was observed from simulations that with a corner radius of less than $2 \mathrm{~mm}$, the return loss would increase by no more than $1 \mathrm{~dB}$ and the frequency shift by less than $5 \mathrm{MHz}$. This, alongside other discrepancies that would result from fabrication tolerance, normally can be corrected by postfabrication tuning. The design $\mathrm{B}$ has been fabricated for verification. The design $\mathrm{A}$ has also been optimized to a return loss level of $19 \mathrm{~dB}$ but not fabricated.

In addition, a two-way power divider with a third order Chebyshev response was also designed. Its responses are compared with the four-way divider in Fig. 5. All the expected poles are clearly seen. The increased selectivity of the fourway divider is evident.

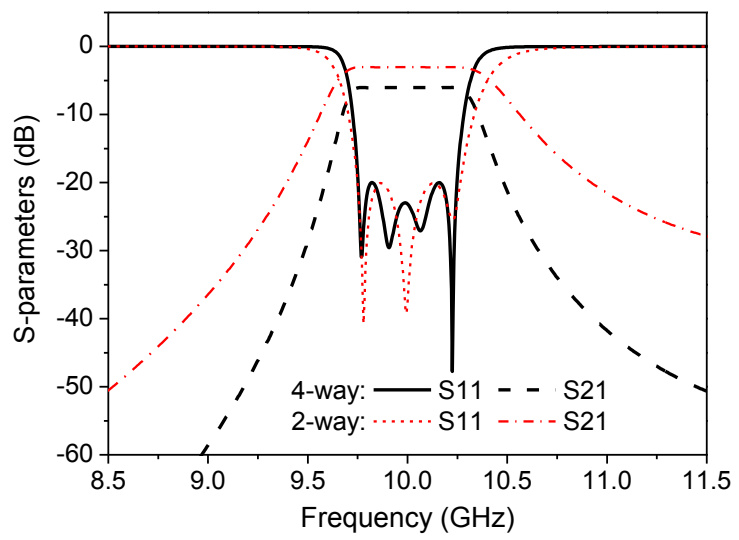

Fig. 5. Comparison between the third-order two-way power divider and the fourth-order four-way divider.

\section{MEASUREMENTS}

The design $\mathrm{B}$ has been fabricated using aluminium as shown in Fig. 6. A two-port network analyser was used to measure the device with the two unused ports terminated by waveguide loads. Fig. 7 shows the measurement results after tuning. Reasonably good agreements between simulations and measurements have been achieved. The bandwidth as defined by the $20 \mathrm{~dB}$ return loss is from $9.71 \mathrm{GHz}$ to $10.20 \mathrm{GHz}$. There is a deviation of $45 \mathrm{MHz}$ which was not corrected by the tuning screws. The noticeable over-machining of the inner waveguide channels is the main contribution of this frequency 
shift. The corner radius is $1.6 \mathrm{~mm}$. According to simulations, this should not affect the frequency by more than $5 \mathrm{MHz}$. The passband transmission is between $-6.7 \mathrm{~dB}$ and $-6.3 \mathrm{~dB}$. This constitutes a loss of $0.3 \mathrm{~dB}-0.7 \mathrm{~dB}$ per channel which is slightly larger than expected. This may be partly a result of non-ideal surface quality. The loosely secured metal tuning screws should also have some contribute to the higher-thanexpected power dissipation.

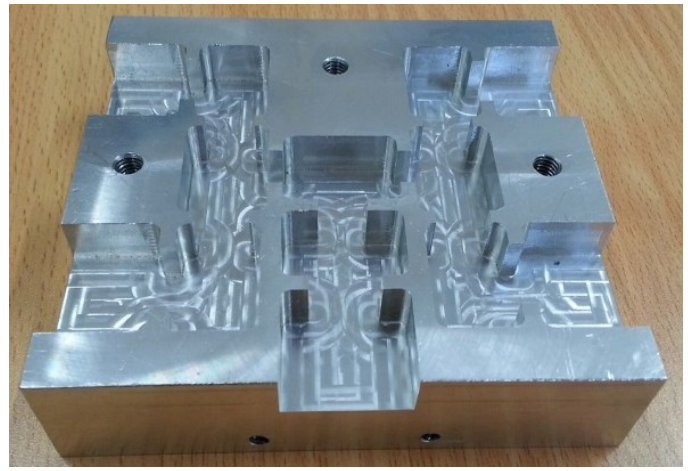

Fig. 6. Photo of the fabricated Design B. The cover including the tuning mechanism is removed to assist with viewing.

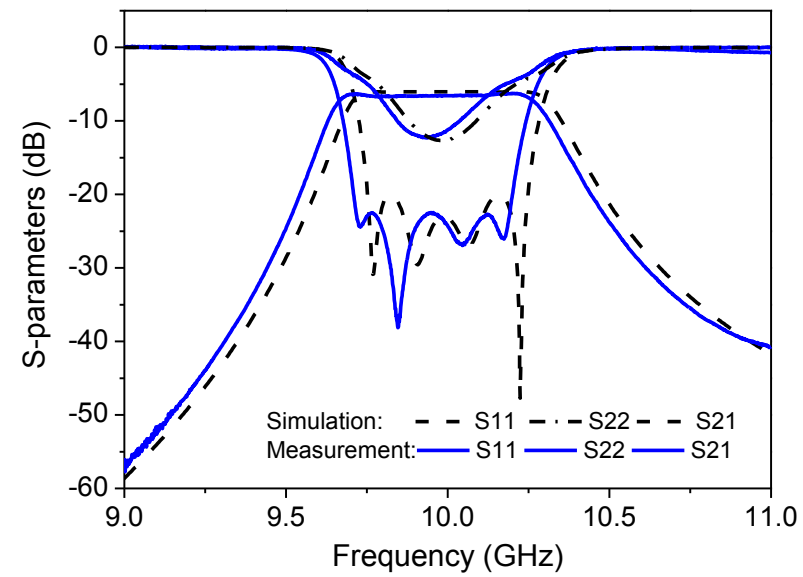

Fig. 7. Measurement results after tuning (solid lines) in comparison with simulations (dash lines).

\section{CONCLUSION}

This paper presented a four-way filtering power divider based on a coupled-resonator network. The coupling matrix of a bifurcated topology with eight resonators was synthesised. Due to the high-symmetry of the equal divider, a direct synthesis is possible. For an arbitrary topology, an optimisation based synthesis method can be used. Two different layouts were designed using X-band rectangular waveguides cavities. Fourth-order Chebyshev responses were obtained from the filtering power divider. The simulated responses showed excellent agreement with the theoretical ones. These were also verified by measurements. Techniques to improve the output matching and the isolation performance between the output ports were not looked at in this paper and will be addressed in the future work. Further work is under way to investigate the bandwidth capabilities of the multi-port coupled-resonator networks.

\section{ACKNOWLEDGMENT}

A. M. M. would like to acknowledge National Information Technology Development Agency (NITDA), Nigeria for providing the MSc scholarship, and the Department of Electrical and Electronic Engineering Technology, The Federal Polytechnic Damaturu, Nigeria for kind financial support. The authors also thank Dr. Xiaobang Shang, the University of Birmingham for helpful discussion.

\section{REFERENCES}

[1] M. Beldi, F. Boone, D. Deslandes, "Design of microstrip power dividers with filtering functions," in 2012 European Microwave Conference, pp. 384-387, Oct. 2012

[2] Y. C. Li, Q. Xue and X. Y. Zhang, "Single and Dual-Band Power Dividers Integrated with Bandpass Filters," IEEE Trans. Microw. Theo. Tech., vol. 61, no. 1, pp. 69-76, 2013.

[3] X. Y. Zhang, K.-X. Wang, B.-J. Hu, "Compact Filtering Power Divider with Enhanced Second-Harmonic Suppression," IEEE Microw. Wireless Comp. Lett., vol. 23, no. 9, pp. 483-485, 2013.

[4] C.-F. Chen, T.-Y. Huang, T.-M. Shen, R.-B. Wu, "Design of Miniaturized Filtering Power Dividers for System-in-a-Package," IEEE Trans. Comp. Package Manuf. Tech., vol. 3, no. 10, pp. 1663-1672, 2013.

[5] T. Shaik, M. Lancaster, F. Huang, "Synthesis of Multiple Output Coupled-Resonator Circuit Using Coupling Matrix Optimasation," IET Microw. Anten. Prop., vol. 5, no. 9, pp. 1081-1088, 2010.

[6] W.-R. Liu, T.-Y. Huang, C.-F. Chen, T.-M. Shen, R.-B. Wu, "Design of a 180-Degree Hybrid with Chebyshev Filtering Response Using Coupled Resonators," 2013 IEEE MTT-S Int. Microwave Symp. Dig., 2013.

[7] L.-S. Wu, Y.-X. Guo and J.-F. Mao, "Balanced-to-Balanced Gysel Power Divider with Bandpass Filtering Response," IEEE Trans. Microw. Theo. Tech., vol. 61, no. 12, pp. 4052-4061, 2013.

[8] K. X. Wang, X. Y. Zhang, B.-J. Hu, "Gysel Power Divider with Arbitrary Power Ratios and Filtering Responses using Coupling Structure," IEEE Trans. Microw. Theo. Tech., vol. 62, no. 3, pp. 431-440, 2014.

[9] X. Shang, Y. Wang, W. Xia and M. J. Lancaster, "Novel Multiplexer Topologies Based on All-Resonator Structures," IEEE Trans. Microw. Theo. Tech., vol. 61, no. 11, pp. 3838-3845, 2013.

[10] G. Macchiarella, S. Tamiazzo, "Synthesis of Star - Junction Multiplexers," IEEE Trans. Microw. Theo. Tech., vol. 58, no. 12, pp. 3732-3741, 2010

[11] CST Microwave Studio. Darmstadt, Germany, CST GmbH, 2013.

[12] J. S. Hong, M. J. Lancaster, Coupled Resonator Circuits in Microstrip Filters for RF/Microwave Applications, New York, John Wiley \& Sons, 2001. 